\title{
Adjustment for Baseline Level of Dengue Cases Due to Increased Testing in Singapore
}

\author{
Li Wei Ang*, Cindy Thiow Koon Lim, Stefan Ma, Joanne Tay and Jeffery Cutter \\ Mlnistry of Health, Singapore
}

\section{Objective}

To make adjustment of historical trends to accurately reflect the baseline level of dengue cases in Singapore, in view of increased testing for dengue in 2013.

\section{Introduction}

Dengue is endemic in Singapore, with epidemics of increasing magnitude occurring on a six-year cycle in 1986/7, 1992, 1998, 2004/5, 2007 and 2013. The incidence per 100,000 population ranged from 87.2 to 105.6 in $2009-2012^{1}$, and surged to 410.6 in 2013 . The mean weekly number of dengue cases over a five-year period provides an indication of the baseline level. We illustrate an adjustment that has been made to the computation of the baseline level due to increased testing for dengue in 2013.

\section{Methods}

Dengue is a legally notifiable disease in Singapore. In any given year, a subset of dengue infected cases is laboratory-confirmed and notified to the Ministry of Health, Singapore. The remaining cases may not be tested as the medical practitioner has clinically diagnosed them to have dengue based on their presenting signs and symptoms, or they may have mild symptoms or are asymptomatic and are not tested for dengue. In 2013, some of these mild, asymptomatic or clinically diagnosed cases may have been "uncovered" and laboratoryconfirmed, due to increased testing for dengue in that year (Fig. 1).

\section{Adjustment factor}

We assumed that the proportion of hospitalizations among laboratory-confirmed dengue cases remained constant between 2012 and 2013. The proportion hospitalized was 0.40 in 2012.

Assuming there was no increased testing and no change in dengue severity, the proportion hospitalized in 2013 should remain the same at 0.40 . Hence, the expected number of notified laboratory-confirmed dengue cases in each week could be derived by dividing the weekly number of hospital admissions by 0.40 . Any increase in observed numbers over the expected numbers would represent an adjustment due to increased testing. As such, the weekly adjustment factor due to increased testing for each week could be derived by dividing the observed weekly number of cases by the expected weekly number.

We also assumed that dengue testing practices had stabilized since epidemiological week (E-week) 29, from 14 to 20 July 2013. The average 4-weekly moving adjustment factor from E-week 29 to E-week 52 in 2013 was 1.54

Removal of outliers

Weekly number of cases which exceeded mean +2 standard deviations (SD) for each of the four years from 2009 to 2012 were deemed as outliers and removed. For 2013, outliers exceeding $1.54 \mathrm{x}$ (mean $+2 \mathrm{SD})$ were removed.

Computation of baseline levels for 2014

The adjustment factor of 1.54 was applied to the remaining weekly data from 2009 to 2012 after outliers had been removed. For 2014, the mean was then computed using the adjusted weekly numbers from 2009 to 2012 and remaining data points in 2013.

\section{Results}

For 2013, the previous mean reflecting the baseline level of dengue was 97 , which was computed based on weekly numbers of dengue cases over a five-year period from 2008 to 2012.

For 2014, the baseline level of dengue with adjustment for increased laboratory testing was higher at 143 cases per week.

\section{Conclusions}

When there are changes to existing public health surveillance such as increased testing for dengue in 2013, there is a need to make adjustment to the computation of baseline level so as to better reflect the underlying disease trends. The mean for 2014 would be lower if the adjustment factor to inflate the weekly numbers from 2009 to 2012 was not incorporated, which would then result in an underestimation of the baseline level for 2014.

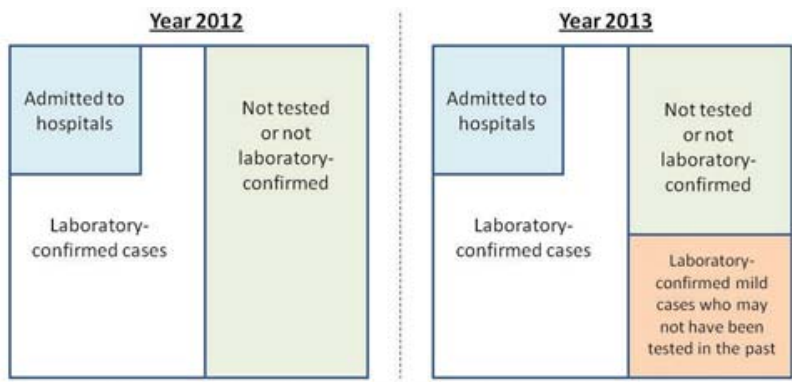

Fig. 1. Pictorial representation of the effect of increased testing of dengue cases in 2013

\section{Keywords}

dengue; endemic; epidemic; laboratory-confirmed; baseline

\section{References}

1. Ministry of Health, Singapore. Communicable Diseases Surveillance in Singapore 2012. Singapore, 2013.

\section{*Li Wei Ang}

E-mail: ang_li_wei@moh.gov.sg 